\title{
Diversity of close-in planets and the interactions with their host stars
}

\author{
Douglas N.C. Lin $^{1,2}$ and Ian Dobbs-Dixon ${ }^{3}$ \\ ${ }^{1}$ Department of Astronomy and Astrophysics, University of California, Santa Cruz, CA, USA \\ email: lin@ucolick.org \\ ${ }^{2}$ Kavli Institute of Astronomy \& Astrophysics, Peking University, Beijing, China \\ ${ }^{3}$ Department of Physics, McGill University, Montreal, Canada \\ email: iandd@physics.mcgill.ca
}

\begin{abstract}
Short period planets provide an exciting opportunity of constraining structural properties. Observations have revealed a diverse class of objects, including several at odds with aspects of conventional planet formation theories. Here we present several scenarios that may help in producing the observed diversity. For short period planets in particular, their proximity to their host stars suggests that star-planet interactions may play an important role in their orbital and structural evolution. We first show that the penetration of a non-synchronous stellar magnetic field into short period planets will provide a significant source of energy for planetary expansion and may help stall inward migration. In addition to magnetic dissipation, the intense irradiation from the host star will drive atmospheric flows, whose behaviour is strongly influenced by the opacity of the envelope. Finally, we explore the role of late stage planetesimal and embryo bombardment on the structure of gas-giant planets. Dynamical trapping during migration, followed by orbital destablization during the final stage of gas-giant growth, leads to a surge in the collision rate. Such collisions will lead to preferential core growth and inflated radii. All three of these processes, occurring late in the planetary formation process, will produce a large range in planetary properties and may account for the diversity we see today.
\end{abstract}

\section{Introduction}

The unanticipated discovery of the first close-in planet around 51 (Mayor \& Queloz 1995) has rekindled the notion that shortly after their formation beyond the snow line some fraction of planets may have migrated to the proximity of their host stars through their tidal interactions with their nascent disks (Lin et al. 1996). After a decade of discoveries, nearly $20 \%$ of the 250 known planets have similarly short periods (Marcy et al. 2005), with approximately one tenth with periods less than 4 days. The short periods imply that their host stars will play an important role in their evolution and appearance. Here we touch on several aspects of that interaction, including 1) a "pile up" in the period distribution at 2-3 days, 2) a large dispersion in the mass-radius distribution of close-in planets, and 3) a diversity in the planetary atmospheric properties.

\section{Magnetospheric Interactions in Short Period Planets}

According to the conventional sequential accretion scenario, planets originally form in the outer regions of the disk, and some fraction of them migrate inward toward their host stars. If these planets indeed migrated to their present-day location, their survival requires a halting mechanism in the proximity of their host stars. Two basic physical 
effects were suggested as potential migration barriers. The first is due to gravitational tidal interactions between the host star and the planet. For tidal frequencies smaller than twice the spin frequency, inertial waves are excited in the convective envelope of the host star and then dissipated locally by turbulent viscosity (Ogilvie \& Lin 2007). However, the tide excited by a close-in gas giant planet in a star with a structure similar to that of the present Sun marginally fails to achieve nonlinearity such that their survival is ensured. Nevertheless, during the formation epoch of solar-type stars, conditions at the center of the star evolve, so that nonlinearity may set in at a critical age, resulting in a relatively intense star-planet tidal interaction.

The second effect suggested is based on the magnetic interaction between the host star and the planet. Young T Tauri stars have radii $\left(R_{*}\right)$ 2-3 times that of the presentday Sun $\left(R_{\odot}\right)$ and fields of several thousand gauss $\left(B_{*}\right)$ on their surface (Johns-Krull 2007). The stellar magnetosphere threads across the inner regions of the disk and clears a cavity out to a critical radius $\left(R_{c}\right)$ which is determined by both the magnitude $B_{*}$ and $\dot{M}_{d}$ (Koenigl 1991). The complex interplay between accretion and outflow leads to angular momentum exchange which induces $\Omega_{*}$ to evolve toward the Keplarian value $\left(\Omega_{k}\right)$ at $R_{c}$. When the planet's orbital semi major axis $(a)$ reduces well inside $R_{c}$, its Lindblad resonances relocate inside the star's magnetospheric cavity. In principle, the planet's migration would stall due to its diminishing tidal torque on the disk.

However, if the star's magnetospheric interaction with the disk can lead to $\Omega_{*}=$ $\Omega_{k}\left(R_{c}\right)$, the planet inside the magnetospheric cavity would have $\Omega_{k}>\Omega_{*}$. In this limit, the star-planet gravitational tidal interaction would induce a transfer of angular momentum from the planet to the star. In addition, the differential motion between the planet and the spinning stellar magnetosphere induces an electromagnetic field with a potential to generate a large current analogous to the interaction between Jovian magnetosphere with its satellite Io (Goldreich \& Lynden-Bell 1969). The associated Lorentz force drives an orbital evolution toward a synchronous state, in which case, angular momentum would be transferred from the planets to their host stars, and the planets would continue their orbital decay. However, a modest resistance in planet will distort the field and may lead to field slippage (Dermott 1970). In the context of interacting binary stars, Campbell (1983) considered the penetration and dissipation of a periodically variable field, associated with an asynchronously spinning primary. In this analysis, Campbell focussed on the flow in the envelope of the secondary and neglected the possibility of current flowing through the flux tube between the secondary star/satellite and the surface of the primary star/planet. This vacuum-surrounding approximation is justifiable since conductivity on the primary is likely to be much larger than that on the secondary and the stationary component of the field is frozen in the white-dwarf primary but not in the main-sequence secondary.

Using a similar approach, Laine \& Lin (2008) carried out an analysis of close-in extra solar planets around young stars with intense magnetic fields. In this investigation, we determine the modulation of the planetary response to the tilted magnetic field of a non-synchronously spinning star. We first compute the conductivity in the interior of young planets, with which we are able to compute the energy dissipation rate inside the planet due to the current induced by the star's magnetic field. The energy dissipation rate is insensitive to changes in the amplitude of the conductivity by several orders of magnitude. A high conductivity increases the energy dissipation in a given volume, but it also tends to prevent the magnetic field from penetrating inside the planet. On the other hand, a lower conductivity corresponds to less dissipation per unit of volume, but it also allows the field to penetrate deeper inside the planet (and therefore increasing the volume where energy can be dissipated). 
The induced current is due to the diffusion of the time dependent magnetic field, coming from the relative motion of the planet's orbit and the stellar magnetosphere. Thus, the ohmic dissipation must be supplied by the orbital kinetic energy of the planet and the rotational energy of the star. Outside corotation, the rotational energy of the star is transferred to the total orbital energy of the planet and provides a supply for the ohmic dissipation. In systems, such as GW Hydra (Setiawan et al. 2008), the migration of the planet may be halted by magnetic coupling with the rapidly spinning magnetised host stars. However, this condition is satisfied only in disks with low gas accretion rates around a rapidly spinning and weakly magnetised stars. A large fraction of T Tauri stars spin with periods longer than those of the close-in planets' orbits ( $\sim 3$ days). Inside corotation the associated torque actually induces the protoplanets to migrate inward. Furthermore, the orbital decay is a runaway process because for a dipolar stellar field the strength of this magnetic interaction decreases more rapidly with semi major axis than that needed for a planet to migrate with a constant speed.

The ohmic dissipation is likely to increase the temperature, the ionization fraction, and the conductivity around the region where most of the dissipation occurs. Increases in the ionization rate modify the skin depth and relocates the region of maximum ohmic dissipation. The heat released by the dissipation is comparable to that associated with the Kelvin-Helmholtz contraction during the early stage of the planet's evolution (Bodenheimer et al. 2001). In the proximity of its host star, this extra energy source may cause a planet to inflate beyond its Hill's radius and lose mass ( $\mathrm{Gu}$ et al. 2004). The tidal (gravitational) interaction between the star and the planet can also significantly enhance the planet's inflation in some cases, particularly for small semi-major axis (the tidal effect varies as $a^{-13 / 2}$ ) and for large radius (the more inflated the planet, the stronger this effect).

Despite this additional source of thermal energy, the envelope of close-in planets is characterised by a core, a convective interior and a radiative atmosphere. The transition between the interior and the atmosphere is generally above the skin depth because only in the convective interior are the temperature, ionization fraction, and conductivity sufficiently large to halt the penetration of the field. In a hydrostatic equilibrium, the actual location is determined by the ratio of the ohmic dissipation rate in the convective region to the sum of the ohmic dissipation rate in the entire planet's interior and the stellar irradiative flux on the planet's surface. A self-consistent solution requires the matching of the ohmic dissipation rate expected from the planetary structure to that which determines its density and temperature distribution.

At distances closer than 0.04 AU around T Tauri stars with kilogauss dipolar fields, the ohmic dissipation is sufficiently intense that the generated heat cannot be adequately transported by convection and radiative diffusion alone. As the internal pressure builds, a fraction of the heat is also transported by advection associated with a slowly expanding envelope. Nevertheless, a state of quasi hydrostatic equilibrium is maintained (Laine \& Lin 2008). For illustration purpose, we computed the internal structure and dissipation rate inside a $0.63 M_{J}$ planet with a semi major axis at $0.04 \mathrm{AU}$ orbiting around a Solar type star that has a magnetic dipole moment $4 \times 10^{34} \mathrm{Am}^{2}$ with a relative angular frequency of $10^{-5} s^{-1}$. In Fig. 1, we plot the Ohmic dissipation rate, mass loss rate, and time scale $(\tau)$ for different stellar irradiative luminosity (in SI units). The magnitude of $\tau$ in this model is comparable to the disk depletion time scale of several Myr.

As a planets' envelope expands to its Roche lobe, it loses mass primarily through the inner Lagrangian point. As mass is transfered from the planet to its host star it also provides angular momentum to the planets ( $\mathrm{Gu}$ et al. 2004) which leads to the expansion of their semi major axis at a rate $\dot{a}=2 a / \tau$. Thus, the resulting mass transfer not only 


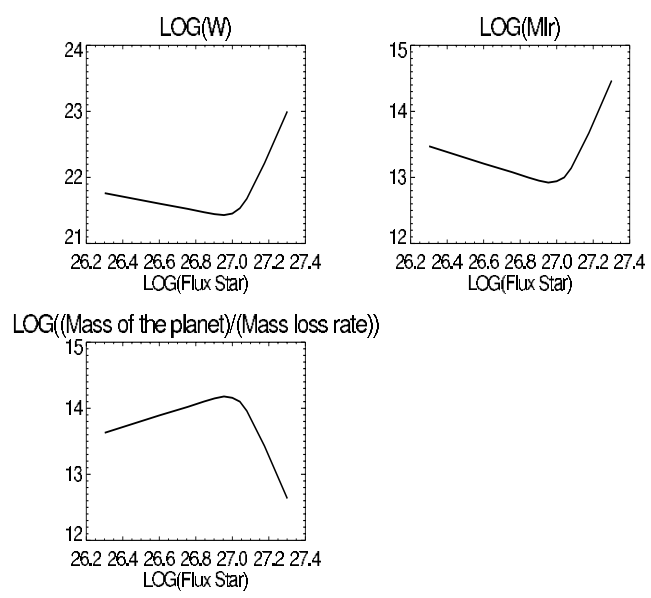

Figure 1. Ohmic dissipation rate $(W)$, mass loss rate (Mlr) and time scale $\tau$ for different stellar irradiative luminosity (in SI units). The magnitude of $\tau$ in this model is comparable to the disk depletion time scale of several Myr.

reduces the planets' mass but also stalls their orbital migration. This process provides a natural explanation for the low medium mass of the close-in planets, in comparison with that of intermediate and long-period planets.

\section{Internal Structure Diversity}

In the conventional planet-formation scenario (Pollack et al. 1996) heavy elements first coagulate into planetesimals and protoplanetary embryos. When they attain a critical mass $M_{\text {crit }} \sim$ a few $M_{\oplus}$ they begin to accrete gas efficiently and to evolve into gas giants. Perhaps the strongest observational support for this scenario is the inferential discovery of cores and super solar metallicity in the envelopes of solar-system gas and ice giants (Guillot et al. 2004). Since the emergence of massive planets is preceded by the formation of Earth-like embryos, the discovery of over 250 extra solar Jupiter-mass planets offers promising circumstantial evidences for the existence of terrestrial planets.

Unfortunately, the present version of the sequential accretion scenario encounters two main challenges: 1) the time scale required for gas giants to form needs to be less than that associated with the depletion of gas in the disk (a few Myr) and 2) the diversity of internal structure and chemical composition among the known gas giants appears to be inconsistent with the expectation of some well defined critical conditions for the onset of gas accretion. The first issue is primarily regulated by a heat transfer inefficiency which suppresses the onset of gas accretion until the cores have attained $3-10 M_{\oplus}$. In the first models this barrier would delay the formation of Jupiter in a minimum mass nebula to 10-20 Myr. There have been several attempts to resolve this paradox. Although this issue is not the central focus of our discussion here, many proposals for the resolution of the accretion-time-scale problem have implications which exacerbate the second paradox which is associated with the gas giants' structural diversity. If the internal structure of the mature planets is assumed to be the relic of some critical conditions that led to their formation, the large observed dispersion would imply many possible paths for their final assemblage. 

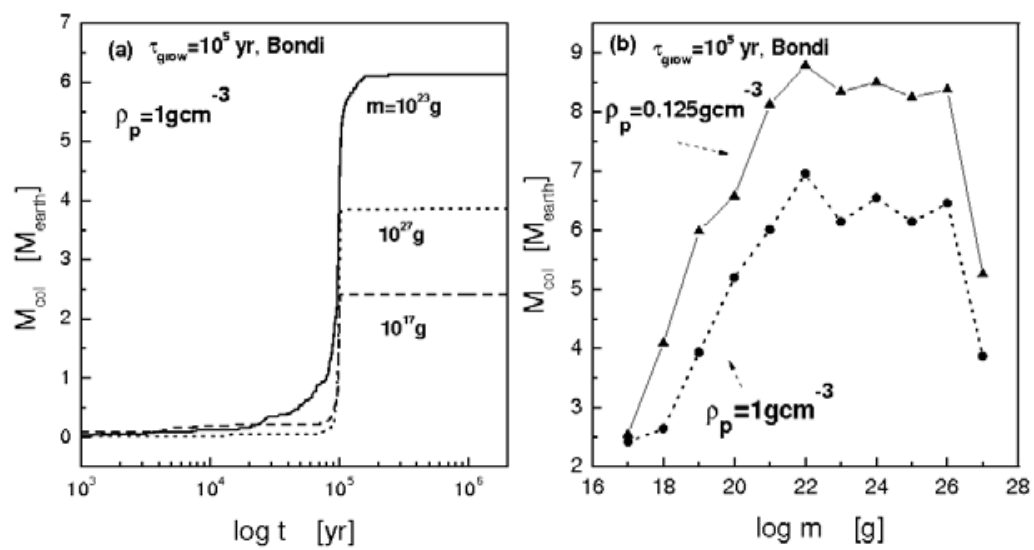

Figure 2. Dependence of collision rate on planetesimal mass (m). (a) Evolution of the total solid mass collided onto the protoplanet with individual planetesimal mass ranging from $10^{17}$ to $10^{27} \mathrm{~g}$ in the Bondi gas-accretion model of $\tau_{\text {grow }}=10^{5} \mathrm{yr}$. (b) Dependence of the collided planetesimals' mass on their individual masses.

The second outstanding problem has been considered in terms of a giant-impact and merger hypothesis, in which the structural and compositional diversity among the gas giants and extrasolar planets is assumed to be the consequence of some catastrophic events. The most vulnerable stage for these collision events is during the build up of the planets' mass. After the cores have attained their isolation masses, additional mass gain through gas accretion enlarges their feeding zone and brings a fresh supply of planetesimals. However, the relatively low-mass cores have limited influence in exciting the newly captured planetesimals' eccentricities. Due to their aerodynamic and tidal interaction with the disk gas, these eccentric planetesimals also undergo slow orbital decay. These planetesimals generally cannot pass through the cores' mean motion resonances and the suppression of their bombardment rate enables the cores to accrete gas with little interruption. However, during the protoplanets' growth, their resonances' widths increase with mass. Once the resonances overlap, the trapped planetesimals become dynamically unstable and their eccentricity excitation is strongly enhanced. Subsequent gas drag induces the planetesimals to migrate to the proximity of the protoplanets and to collide with them. The distance between the protoplanets and their closest separable resonances increases with their mass. This process leads to a surge of planetesimals' bombardment during the advanced stage of protoplanets' growth.

\subsection{Bombardment rate}

To understand the effect of planetesimal and embryo bombardment on the internal structure of a protoplanet we must first calculate the rate of collisions. In this section we present two such calculations. We first consider the destabilising effect of a growing protoplanet on a swarm of planetesimals. The second calculation considers the capture of planetesimals into mean motion resonances during giant planet migration, followed by eccentricity excitation and collision as the disk gas is depleted.

In order to illustrate the effect of a growing protoplanet, we carried out a series of simulations in which a population of identical planetesimals is placed within 15 times the final Roche radius from a growing proto gas giant planet. Using various prescriptions and 


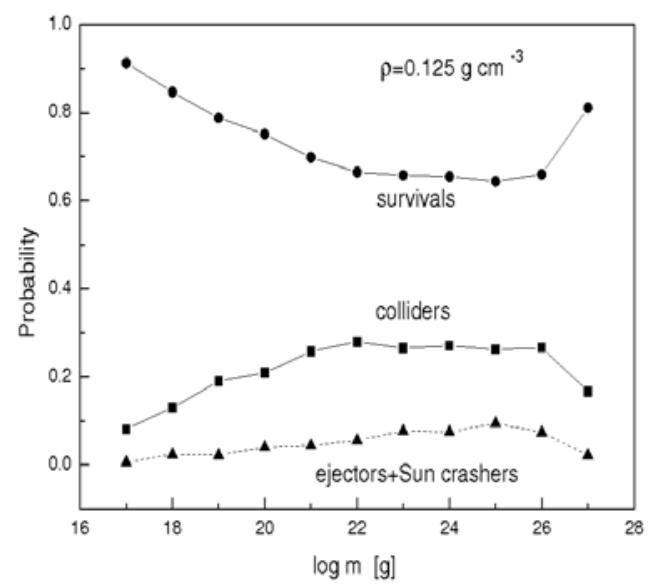

Figure 3. Probability of survivors, colliders, ejectors $(a>100 \mathrm{AU})$, and Sun crashers $(a<1 \mathrm{AU})$ as functions of the planetesimals' mass $\mathrm{m}$ in the range $10^{17}-10^{27} \mathrm{~g}$ at $t=2 \times 10^{6} \mathrm{yr}$. The gas-accretion model for the protoplanet is Bondi accretion. The model parameters are $\tau_{\text {grow }}=10^{5} \mathrm{yr}$ and protoplanet density $\rho=0.125 \mathrm{gcm}^{-3}$.

time scales for the protoplanets mass growth, we show that there is a statistical surge in the planetesimals bombardment rate (Fig. 2). Since they are embedded in their nascent disk, the planetesimals also tidally and hydrodynamically interact with their surrounding gas. In Fig. 3 we plot the probability that planetesimals have either survived, collided with the protoplanet, or been ejected from the system after 2 Myr (when the protoplanet has acquired a Jupiter mass through the conventional Bondi accretion prescription (Zhou \& Lin 2007)). The orbits of the surviving planetesimals are likely to be further destabilised by the emergence of a second-generation gas giant such as Saturn, and will again have a significant probability of crashing into the protoplanet.

The most massive impactors are the residual Earth-mass protoplanetary embryos which may survive the passage through the protoplanets' envelopes and increase the mass of their cores. This effect may also have contributed to the diversity of the core-envelope structures between Jupiter, Saturn and the metallicity dispersion inferred from the transiting extra solar planets. During the final stage of proto-gas-giants' formation, their tidal torque induces the formation of gaps in the disk gas. This perturbed gas-disk structure also leads to the accumulation of planetesimals outside the protoplanets' feeding zone. This surface density enhancement promotes the subsequent build up of cores for secondary gas giant planets outside the orbit of the first-born protoplanets and the formation of eccentric multiple planet systems.

In the core accretion scenario, the growth of protoplanets is stalled when they have swept clean the planetesimals within their feeding-zone of full width $\sim 10-12 R_{h}$, where $R_{h}=\left(M_{e} / 3 M_{*}\right)^{1 / 3} a$ is the Hill's radius. Inside the snow-line of a minimum mass nebular model, embryos acquire an isolation mass $M_{\text {iso }}=0.12(a / \mathrm{AU})^{3 / 4} M_{\oplus}$ on a time scale $\tau_{\text {growth }} \simeq 0.12(a / 1 \mathrm{AU})^{27 / 10} \mathrm{Myr}$ (Ida \& Lin 2004). Outside the snow line, the condensation of ice quadruples the surface density and increases $M_{\text {iso }}$ by an order of magnitude while $\tau_{\text {growth }}$ is reduced by a quarter. The most favourable location for the formation of gas giant planets is therefore across the snow line because particle accumulation can further increase the isolation mass and reduce $\tau_{\text {growth }}$ (see Kretke 2008 and Zhang 2008).

For illustration purpose, we simulate the migration of a Jupiter-mass gas giant formed at $\mathrm{a}=5.2 \mathrm{AU}$ and its capture of residual embryos inside the snow line (Fig. 4). Eccentricity 


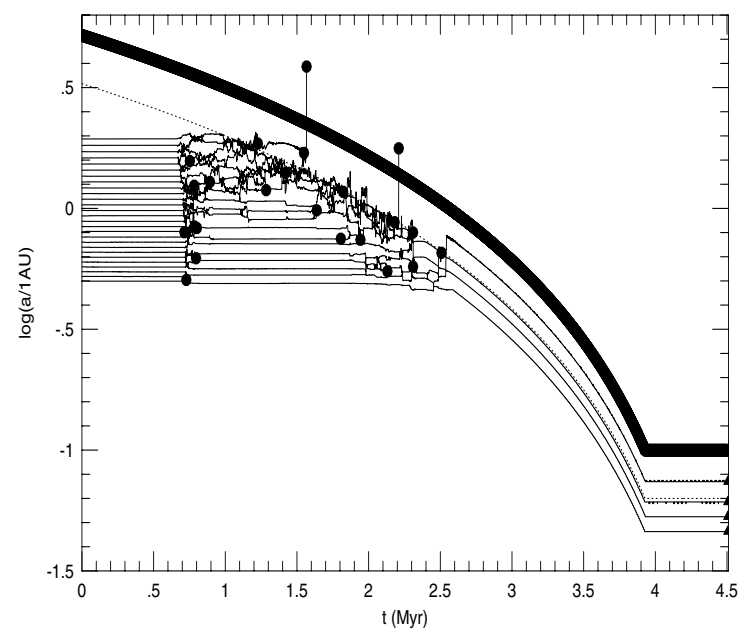

Figure 4. A solitary migrating giant captures residual embryos interior to the snow line onto its MMR. The time evolution of the semi-major axes of the giant and the embryos for model 1 is plotted with heavy and light lines, respectively. The circles and triangles represent collisions and final configurations, and the dotted line shows the location of the 2:1 resonance with the planet. Under the combined influence of e-damping and the planetary resonant perturbation, the captured embryos migrate, collide, and grow.

damping due to the embryos' tidal interaction with the disk is taken into account. The gas giant is assumed to migrate on a time scale of $1.8 \mathrm{Myr}$ and is stalled at $0.1 \mathrm{AU}$. Embryos along the giants' migration path are capture by its mean motion resonances. As a consequence of the embryos' internal tidal dissipation and relativistic precession (Novak et al. 2003; Mardling \& Lin 2004) we anticipate a slight additional semi major axis decay and predict that short-period giants are accompanied by close-in Earths (Zhou et al. 2005).

During the depletion of the disk gas, Jupiter and Saturn's sweeping secular resonances can excite the eccentricity and destabilise the orbits of planetesimals and embryos (Nagasawa \& Lin 2005). After the depletion of the disk gas, long term dynamical instabilities induced by all the gas and ice giant planets on each other can lead to their eccentricity excitation and orbit crossing (Zhou et al. 2007 Zhou Lin). This process widens the planets' feeding zone but reduces their collisional cross section. Eventually, the planets acquire residual planetesimals and embryos well outside their Roche radius.

\subsection{Collisional Simulations}

Since the accretion of the planetesimals and embryos is a stochastic process, it can naturally lead to a wide dispersion in the gas giants $[\mathrm{Fe} / \mathrm{H}]$. Although most of the impacting planetesimals would disintegrate during their passage through the gas giants' envelope, sufficiently massive impinging embryos may reach the cores. For illustration purpose, we carried out two series of simulations ( $\mathrm{Li} \& \mathrm{Lin} 2008$ ). In the first method, we model collisions into gas giant planets using smooth particle hydrodynamics (SPH). It is based on a Lagrangian particle method, and its principle virtues for this study are its ability 
to model highly deformed flows and shocks evolving in three dimensions. Here, we show that direct parabolic collisions generally lead to the total coalescence of impinging gas giants whereas, during glancing collisions, the efficiency of core retention is much larger than that of the envelope. The results of these simulations show that spherical symmetry is quickly reestablished (Fig. 5).
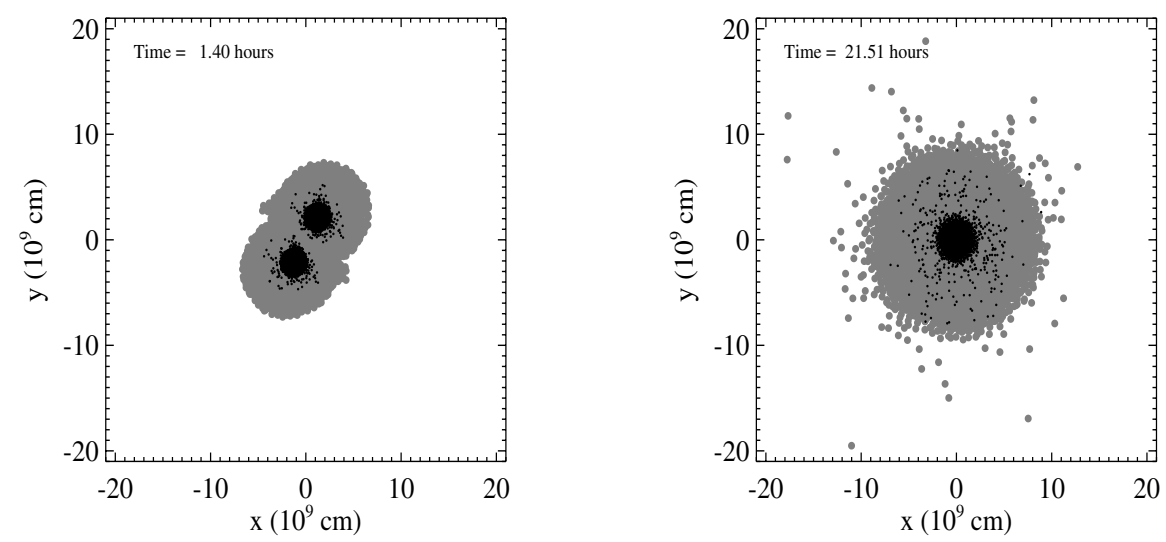

Figure 5. The projection of particles onto the $x-y$ plane during the direct collisions of two gas-giant planets. Iron core material are represented by darker points, while the gaseous envelope is shown in light grey. After about 20 hours (right panel) spherical symmetry has been re-established.

In the limit of negligible rotation, the structural response and evolution of the envelope due to the release of thermal energy during the core coalescence can be computed with a 1D Lagrangian hydrodynamic scheme. In the proximity of their host stars, the expansion of the planets' envelopes (shortly after sufficiently catastrophic impacts) can lead to a substantial loss of gas through Roche-lobe overflow. The accretion of several Earthmass objects can also significantly enlarge the planets' photosphere and elevate the tidal dissipation rate over the time scale of $100 \mathrm{Myr}$. Based on these results, we postulate that the massive core of the compact planet HD149026b is a byproduct of an giant impact event long ago whereas those planets with unusually large radii may have been struck recently by Earth-mass rocky planets.

\section{Atmospheric Heat Flow}

Short period planets receive up to $10^{4}$ times more energy from stellar irradiation then from internal heating. If their negligible eccentricity is a consequence of the tidal dissipation inside these planets, this process would also lead to a state of spin synchronization, with one side perpetually facing their host star (Dobbs-dixon et al. 2004 [cf]). However, the planets' eccentricity may also be damped by tidal dissipation within the host stars, without forcing a state of synchronisation. In either case, the intense stellar irradiation fundamentally modifies the atmospheric dynamics and possibly the internal evolution of these planets. The temperature difference between the day and night sides of the planet is determined by the efficiency of the planet in redistributing energy. If all the stellar irradiated flux is radiated locally, the day-side of the planet would attain an equilibrium temperature $T_{\text {equil }}>1,200 \mathrm{~K}$, while the night-side would cool to an effective temperature 
of $\sim 100 \mathrm{~K}$, comparable to Jupiter. However, if the heat deposited on the day side can be efficiently redistributed, the planet's entire outer envelope would reach an isotropic photospheric temperature of $\sim T_{\text {equil }} / 2^{1 / 4} \sim 10^{3} \mathrm{~K}$. The actual day-night temperature distribution is likely to be between these extreme limits.

Transiting planets are ideal objects for studying planetary atmospheres. Observations include the investigation of primary eclipses with both the Spitzer (Richardson et al. 2006) and the Hubble (Brown et al. 2001) Space Telescopes that reveal crucial information on planetary radii, and IR spectroscopy (Richardson et al. 2003, 2007; Swain et al. 2007; Knutson et al. 2007a) conducted by Spitzer that allows us to probe the composition and thermal structure. In addition, the Spitzer observations of secondary eclipses (Deming et al. 2005; Charbonneau et al. 2005; Deming et al. 2006), and phase monitoring (Harrington et al. 2006; Cowan et al. 2007; Knutson et al. 2007b) allow for the determination of planetary surface temperatures. These observations indicate that at least a fraction of the incident stellar flux is advected from the day to the night side.

Recent observational data also raises several important new puzzles. Among the $>26$ observed radii of transiting extrasolar planets there exist those that are both too large and too small to be explained by conventional formation scenarios. Several solution have been proposed to account for this diversity: 1) the stellar irradiation reduces the temperature gradient and the radiative flux in the planet's outer layers significantly slowing the Kelvin-Helmholtz contraction of the planet (Burrows et al. 2000); 2) a dispersion in the planets' metallicity, opacity, and internal structure may lead to diversity in their mass-radius relation (Burrows et al. 2007a); 3) the kinetic heating resulting from the dissipation of the gas flow within the atmosphere induced by the day-night pressure gradient (Showman \& Guillot 2002); 4) the additional energy provided by tidal dissipation within the planet's envelope (Bodenheimer et al. 2001) caused by the circularization of its orbit may inflate its radius, and 5) the dissipation of the obliquity tides in a Cassinistate resonance between the planet's spin precession and its orbital precession (Winn \& Holman 2005). In each scenario, the efficiency of radiation transfer and atmospheric dynamics play crucial roles.

In this section we present the results of full 3-D, hydrodynamical, radiative transfer models of hot-Jupiter's for a variety of opacities. We cast our numerical model in spherical coordinates and advance the equations of continuity, motion, and energy, including the Coriolis and centrifugal forces. We concentrate on the upper atmosphere $\left(1.0-1.2 R_{J}\right)$ and simulate the azimuth in its entirety. We utilize the flux-limited radiation transfer approximation of Levermore \& Pomraning (1981) to calculate the heat transfer carried by the radiative flux, allowing for a self-consistent treatment of radiation transfer in both the optically thick and optically thin limits. The radiative portion of the energy equation is solved implicitly, while the advection scheme, described in Hawley et al. (1984) and Kley \& Hensler (1987), is an extension of the first-order van Leer (van Leer 1977) method known as the 'mono-scheme'. We use an ideal gas equation of state for the gas pressure and a fixed molecular weight.

For this study we adopted a $0.63 M_{J}$ planet (HD 209458b) that is synchronously spinning with its 3 day orbit around a $4.5 \mathrm{Gyr}$ old solar-type star. In Fig. 6, we illustrate the effective temperature distribution (at the planet's photosphere) of a representative model. For this model, a clear day-night delineation is apparent, with the night-side characterised by temperatures from 270 to $400 \mathrm{~K}$. Slightly hotter regions near the terminators, associated with jets from the day-side, are apparent with temperatures reaching $\sim 480 \mathrm{~K}$. Although the spin period of this planet is much longer than that of Jupiter, the associated Coriolis force alters the resulting flow dynamics and has implications concerning the ability of the planet to fully synchronise its spin. The left-hand plot of Fig. 7 

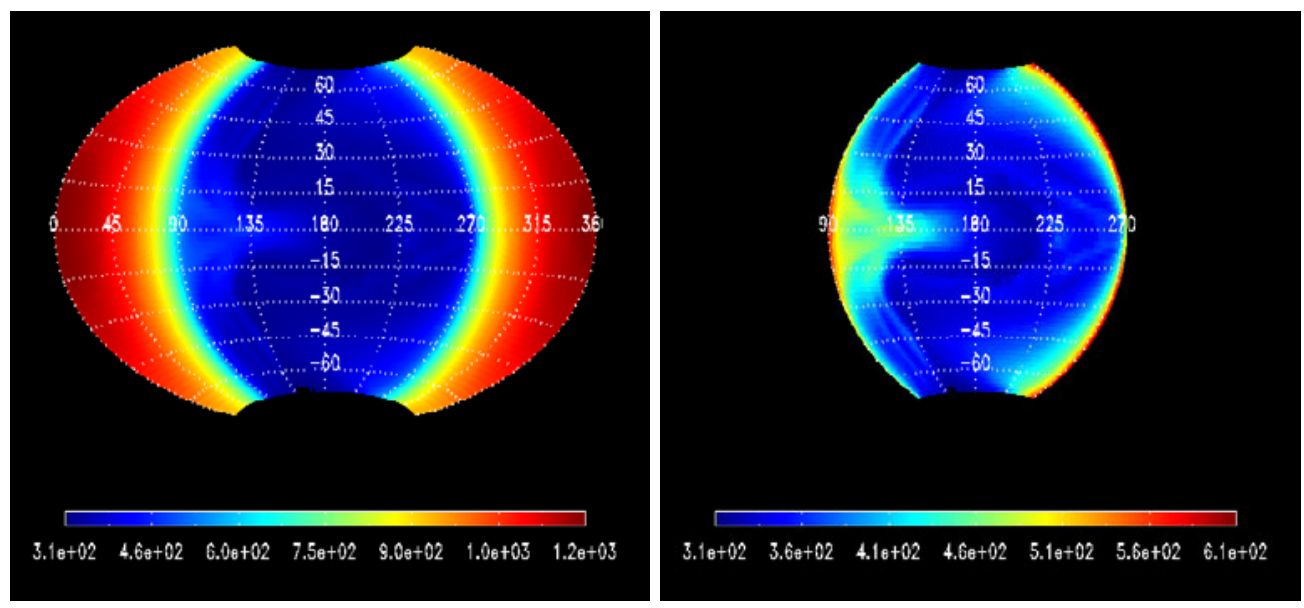

Figure 6. The temperature at the photosphere of a planet rotating with a period of 3days. The left panel shows the entire photosphere, while the right panel highlights the structure on the night-side. Although some higher temperature regions exist associated with jets, a clear day-night delineation persists, despite complicated dynamical structure.
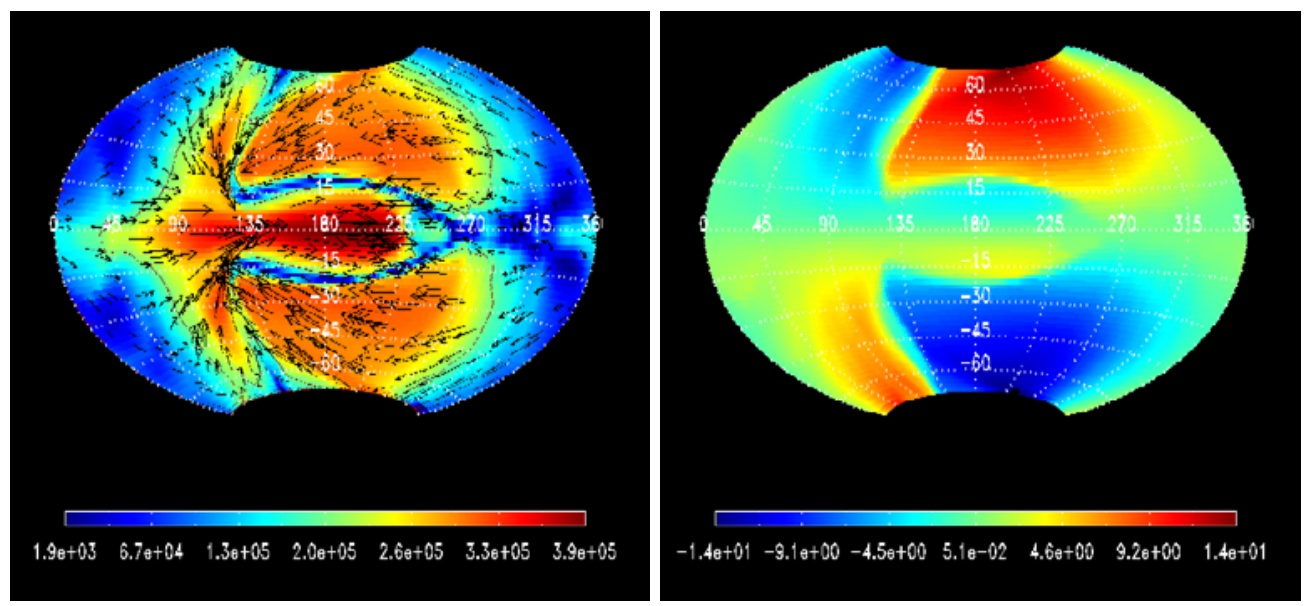

Figure 7. The magnitude of the velocity (left panel) and the latitudinal component of the Coriolis force (right panel) at the photosphere of a planet spinning at 3days. The rotation of the planet causes eastward and westward moving fluid to behave quite differently.

shows the velocity magnitude at the photosphere. Material near the terminator is moving quite rapidly, reaching speeds of over $3 \mathrm{~km} / \mathrm{s}$. Eastward moving material appears to be funnelled into an equatorial jet near $\phi=\frac{\pi}{2}$, while westward moving material is pushed toward the poles near $\phi=\frac{3 \pi}{2}$. The right-hand plot in Fig. 7 shows the latitudinal component of the Coriolis force. The asymmetry imposed by the rotation causes the fluid moving in eastward and westward directions to behave significantly different then in the non-rotating case. These flow structures, and the advected heat they carry account for the structure of the hotter regions seen on the night-side.

Also evident in Fig. 7 is the marked decrease in velocity where the eastward and westward flows converge. It is clear that neither flow is able to instigate circumplanetary flow at the surface. The convergent points, both at the equator and higher latitudes, differ significantly from $\pi$, so upon meeting one of the flows has undergone substantially more 
cooling then its counterpart. Thus, when the eastward flow encounters the westward flow at the equator, it is cooler and sinks below. The opposite is true at higher latitudes, with the westward flow experiencing more cooling and sinking below the eastward flow. The flow pattern is likely to generate potential vorticity and excite baroclinic instabilities. Nevertheless, the dynamics will be dominated by the large scale anisotropic heating imposed by the stellar irradiation.

Our results differ significantly from previous dynamical models (Showman \& Guillot 2002; Cho et al. 2003; Menou et al. 2003; Cooper \& Showman 2005; Cho et al. 2006). These investigations solve either the primitive equations or the equivalent-barotropic equations. The primitive equations are widely used in meteorology and replace the vertical equation of motion with the hydrostatic equation. The equivalent-barotropic equations are derived by vertically integrating the primitive equations. To represent radiative energy transfer, Showman \& Guillot (2002) and Cooper \& Showman (2005) also utilised a Newtonian radiative scheme to approximate stellar irradiation. In this prescription, the temperature is relaxed toward some predefined equilibrium distribution on some radiative timescale. As noted by the authors, this approximation is crude, but allows rapid computation of a large number of models. Our models add two crucial components, including the flux-limited diffusion treatment for radiation and the full 3D treatment. Our results indicate that throughout the entire planet and for a range of opacities the radiative timescale can be quite long in comparison to the dynamical timescale, highlighting the need for a more accurate treatment of radiation. In addition our full 3D simulation flow accurately models the flow in the radial direction. Disallowing this flow would significantly alter the resulting dynamics and leads to much more uniform temperature distributions. In fact, the interpretation (Burrows et al. 2007b) of recent Spitzer observations by (Knutson et al. 2007a) have confirmed a temperature inversion similar to that predicted in ().
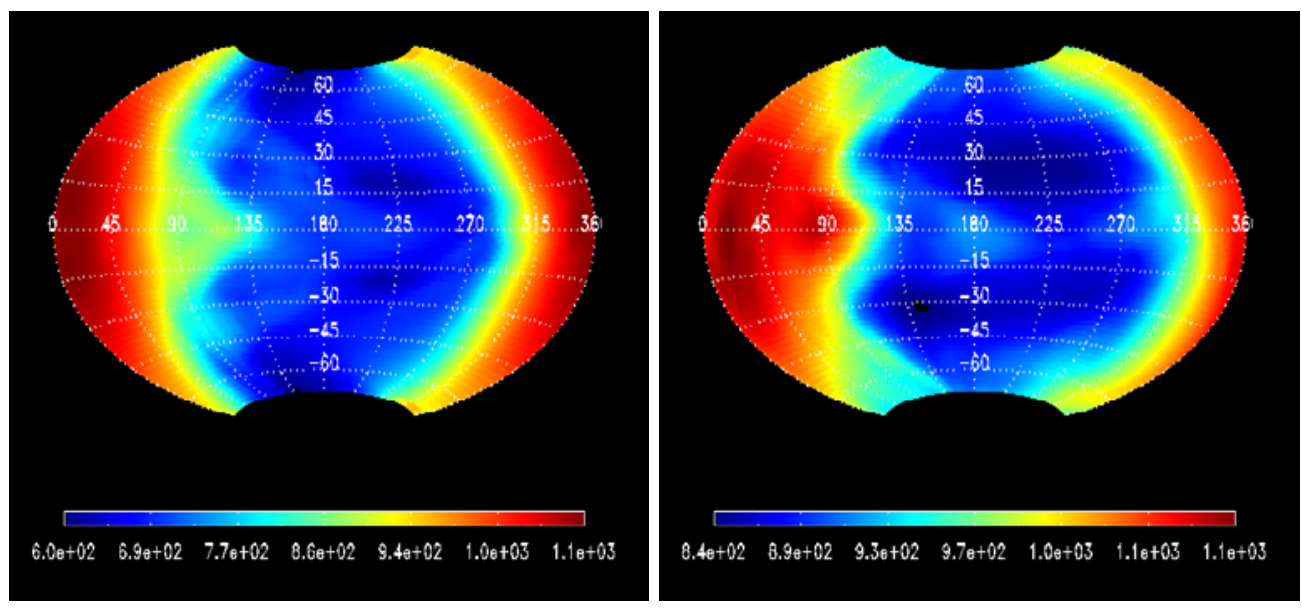

Figure 8. The temperature distribution at the atmosphere of planets whose opacities are reduced by a factor of 100 (left panel) and a factor of 1000 (right panel). Differences in opacity between different planets may play a key role in the observed diversity, and the ability to selfconsistently model the radiative transfer across a wide range in opacity is a crucial component to any dynamical model of these short period planets.

The opacity of the planet regulates the efficiency of both the absorption and reradiation of the incident stellar energy, plays a large role in determining the night-side temperature distribution, and may play a role in the overall evolution of the planetary 
structure. Our current models are based on the interstellar opacities that are most certainly an upper limit to the actual opacities of these atmospheres. Atmospheres containing grains with an abundance and size distribution comparable to that of the interstellar medium will undergo only shallow heating on the day-side; the resulting circulation will not effectively transmit heat to the night-side, which thus cools well below the day-side. However, as the abundance of grains in the atmosphere is reduced, the stellar radiative flux penetrates more deeply into the atmosphere on the day-side, and the higher density atmospheric circulation carries a larger flux of heat over to the night-side. Our numerical simulations show a similar behaviour. In Fig. 8 we show the effects of reducing the atmospheric opacity by factors of 100 (left-hand panel) and 1000 (right-hand panel). In comparison to Fig. 6, the night-side in both these simulations is considerable warmer, reaching $\sim 900 \mathrm{~K}$ for the lowest opacity.

\section{Conclusion}

In this proceeding we have explored several phenomena that may help to explain some of the unusual observed features of short period gas giants. In particular we concentrate on the observed pile-up of planets around 2-3 days, and the large diversity in both structural and atmospheric properties inferred from recent transit observations. The proximity of the host stars suggests that interactions between the star and planet likely play a large role in the evolution of the orbit and the atmosphere.

To explain the pile-up of planets at $2-3$ days we present the results from an analysis of the ohmic dissipation within a planet due to the stellar magnetic field. The magnetic field of young T-Tauri stars may be as large as several kilogauss, which will easily penetrate into the planetary interior to a depth that depends on the planets interior conductivity. A slightly misaligned or non-syncronized magnetic field will lead to field modulation and energy dissipation within the planet. The associated expansion of the envelope with instigate Roche lobe overflow through the inner Lagrange point, providing an outward torque on the orbit. Such interactions ultimately lead to smaller mass planets and stalled migration.

Recent transit observations allow for the computation of mean densities and reveal a large diversity in the structure of gas giants. When compared to the conventional sequential accretion model, there are a number of planets too dense, but also a number that are too large. Such diversity, taken together with the formation constraints imposed by observations of disk lifetimes, suggests that post formation processes may be responsible for the large range in observed properties. We have presented coupled analysis of the rate and nature of planetesimal/embryo bombardment and the resulting structural changes due to the larger of such impacts. SPH simulations show that the angle of giant impacts plays a large role in determining the retention efficiency; head on collisions result in near total assimilation and significant envelope expansion, while in glancing collisions the core of the impactor is preferentially retained perhaps explaining those planets observed to have very large cores.

Finally, continuing observations of close in planets has been revealing a diversity in the atmospheric temperature structures both across the surface of the planet and with depth. We present the results of a three dimensional study of planetary atmospheres irradiated by their host stars. We highlight the importance of using such fully three dimensional radiative hydrodynamical simulation to capture the actual dynamics and energy transfer. In addition we demonstrate the importance of opacity in determining 
the resulting efficiency of heat transport. Changes in opacity due to pollution via methods similar to those described above or the settling/upwelling of high opacity material may help to explain the observed diversity.

\section{References}

Bodenheimer, P., Lin, D. N. C., \& Mardling, R. A. 2001, ApJ, 548, 466

Brown, T. M., Charbonneau, D., Gilliland, R. L., Noyes, R. W., \& Burrows, A. 2001, ApJ, 552, 699

Burrows, A., Guillot, T., Hubbard, W. B., Marley, M. S., Saumon, D., Lunine, J. I., \& Sudarsky, D. 2000, ApJL, 534, L97

Burrows, A., Hubeny, I., Budaj, J., \& Hubbard, W. B. 2007a, ApJ, 661, 502

Burrows, A., Hubeny, I., Budaj, J., Knutson, H. A., \& Charbonneau, D. 2007b, ApJL, 668, L171

Campbell, C. G. 1983, MNRAS, 205, 1031

Charbonneau, D., Allen, L. E., Megeath, S. T., Torres, G., Alonso, R., Brown, T. M., Gilliland, R. L., Latham, D. W., Mandushev, G., O’Donovan, F. T., \& Sozzetti, A. 2005, ApJ, 626, 523

Cho, J. Y.-K., Menou, K., Hansen, B., \& Seager, S. 2006, ArXiv Astrophysics e-prints

Cho, J. Y.-K., Menou, K., Hansen, B. M. S., \& Seager, S. 2003, ApJL, 587, L117

Cooper, C. S., \& Showman, A. P. 2005, ApJL, 629, L45

Cowan, N. B., Agol, E., \& Charbonneau, D. 2007, MNRAS, 552

Deming, D., Harrington, J., Seager, S., \& Richardson, L. J. 2006, ApJ, 644, 560, secondary Ecclipse Discovery Paper for HD189733b

Deming, D., Seager, S., Richardson, L. J., \& Harrington, J. 2005, Nature, 434, 740

Dermott, S. F. 1970, MNRAS, 149, 35

Dobbs-Dixon, I., \& Lin, D. N. C. 2008, ApJ, 673, 513

Dobbs-Dixon, I., Lin, D. N. C., \& Mardling, R. A. 2004, ApJ, 610, 464

Goldreich, P., \& Lynden-Bell, D. 1969, ApJ, 156, 59

Gu, P.-G., Bodenheimer, P. H., \& Lin, D. N. C. 2004, ApJ, 608, 1076

Guillot, T., Stevenson, D. J., Hubbard, W. B., \& Saumon, D. 2004, The interior of Jupiter (Jupiter. The Planet, Satellites and Magnetosphere), 35-57

Harrington, J., Hansen, B. M., Luszcz, S. H., Seager, S., Deming, D., Menou, K., Cho, J. Y.-K., \& Richardson, L. J. 2006, Science, 314, 623

Hawley, J. F., Wilson, J. R., \& Smarr, L. L. 1984, ApJS, 55, 211

Ida, S., \& Lin, D. N. C. 2004, ApJ, 604, 388

Johns-Krull, C. M. 2007, ApJ, 664, 975

Kley, W., \& Hensler, G. 1987, A\&A, 172, 124

Knutson, H. A., Charbonneau, D., Allen, L. E., Burrows, A., \& Megeath, S. T. 2007a, ArXiv e-prints, 709

Knutson, H. A., Charbonneau, D., Allen, L. E., Fortney, J. J., Agol, E., Cowan, N. B., Showman, A. P., Cooper, C. S., \& Megeath, S. T. 2007b, Nature, 447, 183

Kretke, K. A. Lin, D. N. C., Turner, N. J. 2008, in: Y.-S. Sun, S. Ferraz-Mello \& J.-L. Zhou, (eds.), Exoplanets: Detection, Formation and Dynamics, Proc. IAU Symposium No. 249 (Suzhou,China), p. 293

Koenigl, A. 1991, ApJL, 370, L39

Laine, R., \& Lin, D. 2008, in preparation

Levermore, C. D., \& Pomraning, G. C. 1981, ApJ, 248, 321

Li, S., \& Lin, D. 2008, in preparation

Lin, D. N. C., Bodenheimer, P., \& Richardson, D. C. 1996, Nature, 380, 606

Marcy, G., Butler, R. P., Fischer, D., Vogt, S., Wright, J. T., Tinney, C. G., \& Jones, H. R. A. 2005, Progress of Theoretical Physics Supplement, 158, 24

Mardling, R. A., \& Lin, D. N. C. 2004, ApJ, 614, 955

Mayor, M., \& Queloz, D. 1995, Nature, 378, 355

Menou, K., Cho, J. Y.-K., Seager, S., \& Hansen, B. M. S. 2003, ApJL, 587, L113 
Nagasawa, M., \& Lin, D. N. C. 2005, ApJ, 632, 1140

Novak, G. S., Lai, D., \& Lin, D. N. C. 2003, 294, 177

Ogilvie, G. I., \& Lin, D. N. C. 2007, ArXiv Astrophysics e-prints

Pollack, J. B., Hubickyj, O., Bodenheimer, P., Lissauer, J. J., Podolak, M., \& Greenzweig, Y. 1996, Icarus, 124, 62

Richardson, L. J., Deming, D., Horning, K., Seager, S., \& Harrington, J. 2007, ArXiv Astrophysics e-prints

Richardson, L. J., Deming, D., \& Seager, S. 2003, ApJ, 597, 581

Richardson, L. J., Harrington, J., Seager, S., \& Deming, D. 2006, ApJ, 649, 1043

Setiawan, J., Henning, T., Launhardt, R., Muller, A., Weise, P., \& Kurster, M. 2008, Nature, 451,38

Showman, A. P., \& Guillot, T. 2002, A\&A, 385, 166

Swain, M. R., Bouwman, J., Akeson, R., Lawler, S., \& Beichman, C. 2007, ArXiv Astrophysics e-prints

van Leer, B. 1977, Journal of Computational Physics, 23, 276

Winn, J. N., \& Holman, M. J. 2005, ApJL, 628, L159

Zhang, X. J., Kretke, K. A. Lin, D. N. C. 2008, in: Y.-S. Sun, S. Ferraz-Mello \& J. -L. Zhou, (eds.), Exoplanets: Detection, Formation and Dynamics, Proc. IAU Symposium No. 249 (Suzhou,China), p. 309

Zhou, J. -L., Aarseth, S. J., Lin, D. N. C., \& Nagasawa, M. 2005, ApJL, 631, L85

Zhou, J.-L., \& Lin, D. N. C. 2007, ApJ, 666, 447

Zhou, J.-L., Lin, D. N. C., \& Sun, Y.-S. 2007, ApJ, 666, 423 